\title{
One- and two-dimensional Raman induced diffraction gratings in atomic media
}

\author{
V. G. Arkhipkin ${ }^{1,2 *}$ and S. A. Myslivets ${ }^{1,3 \dagger}$ \\ ${ }^{1}$ Kirensky Institute of Physics, Federal Research Center KSC SB RAS, 660036 Krasnoyarsk, Russia \\ ${ }^{2}$ Institute of Nanotechnology, Spectroscopy and Quantum Chemistry, \\ Siberian Federal University, Krasnoyarsk 660041, Russia and \\ ${ }^{3}$ Department of Photonics and Laser Technology, \\ Siberian Federal University, Krasnoyarsk 660041, Russia
}

(Dated:)

\begin{abstract}
We propose and analyze an efficient scheme for the one- and two-dimensional atomic gratings based on periodic spatial modulation of the Raman gain and dispersion, which we name the Raman induced diffraction gratings (RIDGs). There are fundamentally different from those based on electromagnetically induced transparency. As the probe field propagates along the direction normal to the standing pump wave, it can be effectively diffracted into high-order directions. The grating is a superposition of an amplitude and a phase gratings. We identify the conditions when all highorders diffraction are amplified. In addition, we also show that diffraction of a probe field could be dynamically controlled using an additional laser field. With its help, it is possible to suppress or amplify diffraction beams. The RIDGs can be considered as all-optical multi-beam splitters with amplification.
\end{abstract}

PACS numbers: 42.50.Gy, 42.50.Hz, 42.65.Dr, 42.79.Dj

\section{INTRODUCTION}

Electromagnetically induced gratings (EIGs) based on electromagnetically induced transparency (EIT) [1] in a standing coupling field have been studied widely both theoretically and experimentally [2-6]. EIGs stem from periodic spatial modulation of the absorption and/or refractive index of the atoms. Therefore these structures are also referred to as electromagnetically induced absorption gratings (EIAG) [5]. Transmission and reflection spectra of EIG are significantly different from those of a typical EIT configuration. Under certain conditions EIG can have band gaps and transmission bands similar to photonic crystals which can be dynamically tuned [7-9]. Such periodic structures can be useful for all-optical control of light propagation [10-12], biphoton spectrum shaping [4], localization of atoms [13], electromagnetically induced Talbot effect $[14,15]$, one- and twodimensional (1D and 2D) surface solitons in an atomic medium [16]. Applying an additional driving field in a four-level N-type scheme offers further advantages for dynamic control of EIG spectral properties [11, 17].

If a standing wave is perpendicular to the propagation direction of the probe beam, a transverse EIAG can be generated $[2,18,19]$. Such a grating is a diffraction grating. It can diffract a probe beam into higher-order directions, resulting in splitting into several beams. An electromagnetically induced phase grating based on the giant Kerr nonlinearity under EIT [20] in a standingwave signal field has been proposed in $[21,22]$ to improve the first-order diffraction efficiency. Also considered in this scheme are tunable volume transmission holographic

\footnotetext{
*avg@iph.krasn.ru

† sam@iph.krasn.ru
}

gratings [23]. Image-induced blazed gratings based on EIAG were proposed in [24], which can provide new possibilities for all-optical beam splitting and fanning. The authors [25] extended 1D EIAG to the case of two dimensions. However, EIAG produces many diffraction orders with uneven intensity and low efficiency so that it is difficult to control the diffracted beams, which may limit the applications of EIAG. Therefore, seeking new approaches to improve the efficiency of higher diffraction orders is of undoubted interest. In a recent paper [26], the author proposed $1 \mathrm{D}$ and 2D electromagnetically induced polarization diffraction grating based on EIT for realization polarization-dependent multiple beam splitting in the Raman-Nath limit. An electromagnetically induced phase diffraction grating controlled by spontaneous generated coherence (SGC) in atomic systems is studied in $[27,28]$ The possibility of significant improvement of the intensity of higher-order diffractions via relative phase between applied laser fields due to the existence of SGC is shown. The authors [29] propose a gain-phase diffraction grating based on spatial modulation of the crossKerr nonlinearity in a Raman active medium. This grating is induced by a pump field and a standing control field in ultracold atoms. A two-dimensional diffraction grating is demonstrated in a such scheme [30]. Owing to spatially periodic modulation of the probe gain, the diffracted beam in the zero-order direction is amplified, and the higher-order diffraction efficiency is higher than that of EIAG but without amplification.

Recently we have proposed an alternative approach to the generation of EIG based on Raman interaction of the probe field with a standing-wave pump field in three- and four-level media [31-33]. Such gratings are called Raman-induced gratings (RIGs). Unlike EIAG schemes, where absorption is spatially modulated, RIGs are based on spatial modulation of the Raman gain in 


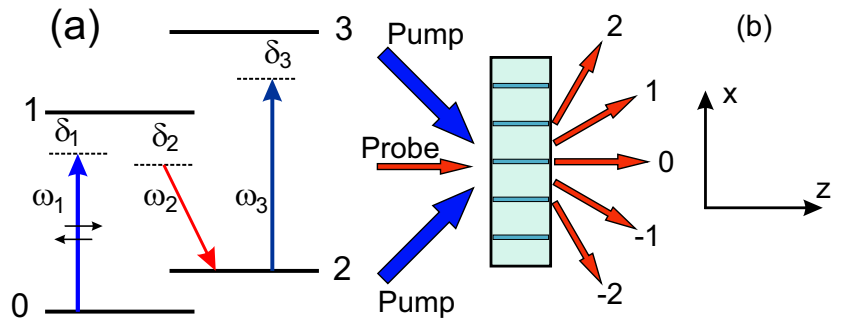

FIG. 1. (a) A four-level N-type atom interacting with three laser beams: standing pump field (with the frequency $\omega_{1}$ ), probe (the frequency $\omega_{2}$ ) and coupling (the frequency $\omega_{3}$ ). $\delta_{i}(i=1,2,3)$ are one-photon detunings from the respective transitions. The atoms are initially populated in the ground state $|0\rangle$. (b) A sketch of the probe- and pump-beam spatial configuration with respect to the atomic sample and diffraction orders.

a standing-wave pump field. RIG can work as a diffraction grating when the probe field propagates along the direction normal to the standing wave. In this paper, we present $1 \mathrm{D}$ and $2 \mathrm{D}$ diffraction gratings formed in an atomic medium due to Raman interaction of a standingwave pump with a weak probe field propagating perpendicular to the standing wave which is called Raman induced diffraction grating (RIDG). The principal advantage of the proposed scheme is that the probe wave can diffract into many higher-order directions with amplification under certain conditions. The higher-orders diffraction intensity depends on the amplitude of the standingwave pump and the two-photon detuning. The intensity of the diffracted beams can be higher than the intensity of the input beam. Such a grating can be considered as an all-optical multi-beam-splitter with amplification. Using an additional driving field in a four-level N-type atomic system, one can control the intensity of higherorders diffraction by suppressing or amplifying them.

\section{THEORETICAL MODEL AND BASIC EQUATIONS}

\section{A. One-dimensional Raman induced diffraction grating}

First we consider the case of a 1D grating. The atomic system under consideration can be described by a four- level N-type configuration (Fig 1a). A homogeneously broadened medium is assumed. Level $|0\rangle$ is the ground state and $|2\rangle$ is a metastable state. Transitions $|0\rangle-$ $|1\rangle,|2\rangle-|1\rangle$ and $|2\rangle-|3\rangle$ are electric dipole allowed, while transitions $|0\rangle-|2\rangle$, and $|1\rangle-|3\rangle$ are electric dipole forbidden. A standing-wave pump

$$
E_{p}(x)=E_{1} \sin (\pi x / \Lambda) \exp \left(-i \omega_{1} t+i k_{1 z} z\right)
$$

interacts with the transition $|0\rangle-|1\rangle$ with a large detuning $\delta_{1}$. The standing pump wave is the result of two travelling waves (with the amplitude $E_{1}$ ) overlapping at an angle $\Psi$ to each other (Fig.1b), thus forming interference bands in the direction of the $x$ axis with a spatial period $\Lambda=\lambda_{1} /[2 \sin (\Psi / 2)]$, depending on the $\Psi$ angle. The probe (Raman) field $E_{2}=1 / 2 E_{2} \exp \left[-i\left(\omega_{2} t-k_{2} z\right)\right]$ propagates along the $z$ direction normally to the standing wave and interacts with the transition $|2\rangle-|1\rangle$. The control field $E_{3}=1 / 2 E_{3} \exp \left[-i\left(\omega_{3} t-k_{3} z\right)\right]$ propagates in the same direction and interacts with the transition $|2\rangle-|3\rangle$. Here we consider the far-off-resonant Raman transitions, namely, $\delta_{1} \gg \gamma_{10}, G_{1}$, and $\delta_{2} \gg \gamma_{21}$, and assume the probe field to be weak.

Induced polarization at the probe frequency $\omega_{2}$ will be $P\left(\omega_{2}\right)=\chi_{2}\left(\omega_{2}\right) E_{2}$, where $\chi_{2}\left(\omega_{2}\right)$ is the macroscopic Raman susceptibility, $E_{2}$ is the amplitude of the probe field. An expression for $\chi_{2}\left(\omega_{2}\right)$ can be derived from the density matrix equations describing interaction of three fields with four-level atoms (Fig.1) to the first order of the probe field, and all-orders of other fields [29, 33, 34]. In the steady state approximation, we have

$$
\chi_{2}\left(\omega_{2}\right)=-i \alpha_{r} F\left(\omega_{2}\right)
$$

where

$$
F\left(\omega_{2}\right)=\frac{\gamma_{12}}{\Delta_{1}} \frac{\left|G_{p}\right|^{2}\left(\Delta_{30} \Delta_{31}+\left|G_{p}\right|^{2}-\left|G_{3}\right|^{2}\right)}{\left(\left|G_{p}\right|^{2}-\left|G_{3}\right|^{2}\right)^{2}+\left(\Delta_{20}\left|G_{p}\right|^{2}+\Delta_{31}\left|G_{3}\right|^{2}\right) \Delta_{2}^{*}+\left(\Delta_{31}\left|G_{p}\right|^{2}+\Delta_{20}\left|G_{3}\right|^{2}\right) \Delta_{30}+\Delta_{30} \Delta_{20} \Delta_{31} \Delta_{2}^{*}} .
$$

Here $\alpha_{r}=\left|d_{12}\right|^{2} N / 2 \hbar \gamma_{12}, G_{p}(x)=G_{1} \sin (\pi x / \Lambda), G_{1}=$ $E_{1} d_{10} / \hbar, 2 G_{3}=E_{3} d_{32} / \hbar$ are the Rabi frequencies of the pump and control field, $\Delta_{1}=\gamma_{10}-i \delta_{1}, \Delta_{2}=\gamma_{12}-i \delta_{2}$, $\Delta_{3}=\gamma_{32}-i \delta_{3}, \Delta_{30}=\gamma_{30}-i \delta_{30}, \Delta_{31}=\gamma_{31}-i \delta_{31}$, $\delta_{1,2,3}=\omega_{1,2,3}-\omega_{10,12,32}$ are one-photon detunings, $\delta_{20}=$ $\delta_{1}-\delta_{2}$ is the Raman detuning, $\delta_{30}=\delta_{1}-\delta_{2}+\delta_{3}, \delta_{31}=$ $\delta_{3}-\delta_{2}, \omega_{m n}, \gamma_{m n}$ and $d_{m n}$ are the frequency, halfwidth and matrix dipole moment of the respective transitions, 
$\hbar$ is the reduced Plank constant.

It is clear from (2) that the Raman susceptibility is modulated by a standing pump wave with the period $\Lambda$ along the $x$ direction. This leads to spatial modulation of the Raman gain and the refractive index. The Raman susceptibility $\chi_{2}\left(\omega_{2}\right)$ for the probe field can be actively and effectively controlled via adjusting the optical parameters of the system, such as the intensities and detunings of the applied fields. This is due to the ac-Stark shift of the resonance frequency of the Raman transition under the action of the pump and the control field (see Appendix). When a probe wave propagating perpendicular to the standing pump wave, it diffracts on the inhomogeneities of the Raman gain and refractive index generating a series of radiation maxima with various wave vector directions (diffraction orders). We called such a structure as RIDG. It should be emphasized that this grating is a hybrid one: an amplitude (gain) grating and a phase (refraction) one.

In the thin grating approximation, when diffraction in the bulk of the atomic sample can be ignored, propagation of a probe wave in a steady state case is governed by the reduced wave equation [2]

$$
\frac{\partial E_{2}(x, z)}{\partial z}=i F\left(\omega_{2}, x\right) E_{2}(x, z)
$$

where $x$ and $z$ are dimensionless variables given in $\Lambda$ and $z_{0}=1 / k_{2} \alpha_{r}$ units, respectively. Equation (3) assumes that the Fresnel number $N_{F}=4 \pi \Lambda^{2} / \lambda_{2} z_{0}$ of a slit of width $2 \pi^{1 / 2} \Lambda$ at the distance $z_{0}$ satisfies the condition $N_{F} \gg 1[2]$.

By solving Eq. (3), the transmission function $T(x)=$ $E_{2}(z=L) / E_{2}(z=0)$ for the interaction length $L$ of the medium (grating thickness) can be obtained as

$$
\begin{gathered}
T(x)=\exp \left[-\operatorname{Im} F\left(\omega_{2}\right) L\right] \exp \left[i \operatorname{Re} F\left(\omega_{2}\right) L\right] \\
=|T(x)| \exp [i \Phi(x)],
\end{gathered}
$$

where $|T(x)|=\exp \left[-\operatorname{Im} F\left(\omega_{2}\right) L\right]$ and $\Phi(x)=$ $\left.\operatorname{Re} F\left(\omega_{2}\right) L\right][$ are the amplitude and phase of the transmission function $T(x)$. Note that the length $L$ is chosen such that the Fresnel number $N_{F}(z=L)=4 \pi \Lambda^{2} / \lambda_{2} L$ at the distance $L$ is also $N_{F} \gg 1$.

For a plane probe wave, intensity distribution in the far field normalized to $\left(E_{2}(z=0) M\right)^{2}$ (Fraunhofer diffraction) is given by [2]

$$
I_{2}(\theta)=\left|E_{2}^{1}(\theta)\right|^{2} \frac{\sin ^{2}\left(M \pi \Lambda \sin \theta / \lambda_{2}\right)}{M^{2} \sin ^{2}\left(\pi \Lambda \sin \theta / \lambda_{2}\right)}
$$

where $M$ is the number of spatial periods of the grating irradiated by the probe beam, $\theta$ is the diffraction angle with respect to the $z$ direction. The diffraction order $n$ is found as $\sin \theta=n \lambda_{2} / \Lambda, n=0, \pm 1, \pm 2, \ldots, \pm(M-1)$, and $E_{2}^{1}(\theta)=E_{2}(\theta) / E_{2}(z=0)$ is

$$
E_{2}^{1}(\theta)=\int_{0}^{1} T(x) \exp \left(-i 2 \pi \Lambda x \sin \theta / \lambda_{2}\right) d x
$$

describes Fraunhofer diffraction over one spatial period of the grating.

\section{B. Two-dimensional Raman induced diffraction grating}

Now we extended 1D RIDG to the case of two dimensions. For a 2D grating, the pump field is a combination of two orthogonal standing-wave fields of the same frequency, that is

$$
E_{p}(x, y)=E_{1}\left[\sin \left(\pi x / \Lambda_{x}\right)+\sin \left(\pi y / \Lambda_{y}\right)\right]
$$

where $\Lambda_{x}$ and $\Lambda_{y}$ are the standing-wave field spatial periods in the $x$ and $y$ directions, respectively. The probe field propagates along the $z$ direction and passes through the intersection region of the two orthogonal standingwave fields in the $x-y$ plane, while the control field may propagate along an arbitrary direction. In this case, the transmission function $T(x, y)$ is a function of two variables $x$ and $y$. In the thin grating approximation it has the form (5). By Fourier transformation of $T(x, y)$, we can obtain the intensity of Fraunhofer diffraction

$$
I_{2}\left(\theta_{x}, \theta_{y}\right)=\mid E_{2}^{1}\left(\theta_{x},\left.\theta_{y}\right|^{2} \frac{\sin ^{2}\left(M \pi \Lambda_{x} \sin \theta_{x} / \lambda_{2}\right)}{M^{2} \sin ^{2}\left(\pi \Lambda_{x} \sin \theta_{x} / \lambda_{2}\right)} \times \frac{\sin ^{2}\left(N \pi \Lambda_{y} \sin \theta_{y} / \lambda_{2}\right)}{N^{2} \sin ^{2}\left(\pi \Lambda_{y} \sin \theta_{y} / \lambda_{2}\right)}\right.
$$

where

$$
E_{2}^{1}\left(\theta_{x}, \theta_{y}\right)=\int_{0}^{1} \int_{0}^{1} T(x, y) \exp \left(-i 2 \pi x \Lambda_{x} \sin \theta_{x} / \lambda_{2}-i 2 \pi y \Lambda_{y} \sin \theta_{y} / \lambda_{2}\right) d x d y
$$

represents the Fraunhofer diffraction of a single space period, and $\theta_{x}$ and $\theta_{y}$ are the diffraction angles with respect to the $x$ and $y$ axes. The conditions $\sin \theta_{x}^{m}=m \lambda_{2} / \Lambda_{x}$ and $\sin \theta_{y}^{n}=n \lambda_{2} / \Lambda_{y}$ correspond to the position of the main diffraction maxima $I_{2}\left(\theta_{x}^{m}, \theta_{y}^{n}\right) . \quad M$ and $N$ are the numbers of spatial periods along the $x$ and $y$ axes of the grating illuminated by the probe beam.

It should be noted that in the general case the two- 
dimensional diffraction grating is not a simple superposition of two one-dimensional gratings, in contrast to conventional ones. This is due to the fact that the nonlinear susceptibility, and hence the transmission function $T(x, y)$, which determines the spatial structure of the grating, is a non-linear function of the pump field and coordinates (see appendix, equation (A4)) and can not be represented as a product of functions that depend only on one coordinate.

\section{RESULTS AND DISCUSSION}

In this section, the equations outlined in Sec. II are used to investigate the diffraction power of RIDG under various parameters. Our numerical analysis employs data corresponding to the sodium atom D1 line and levels $|0\rangle$ and $|2\rangle$ correspond to the long-lived superfine sublevels of the ground state $3 \mathrm{~S}_{1 / 2}$. The following atomic parameters are used: $\gamma_{10} / 2 \pi=10 \mathrm{MHz}, \gamma_{20}=\gamma_{10} / 1000$. It is assumed that $\gamma_{21}=\gamma_{30}=\gamma_{32}=\gamma_{10}$. The Rabi frequencies and one-photon detunings $\delta_{1,2,3}$ are given in $\gamma_{10}$ units and the Raman detuning $\delta_{20}$ in the units $\gamma_{20} . L$ is given in the units of linear absorption length $z_{0}$. Here, we use $\delta_{1}=-100$, the number of periods $D / \Lambda=M=5$ and $L=40$.

\section{A. 1D RIDG}

We first consider a $1 \mathrm{D}$ grating in the case of zero control field $G_{3}=0$. Typical curves of the amplitude $|T(x)|$ as a function of $x$ are shown in Fig. 2a,c,e for various $G_{1}$ and $\delta_{20}$. In these figures, the nodes are located at both ends of the periods while antinodes are located in the middle. It's clear that the probe field is amplified and the probe gain depends on the transverse coordinate $x$ and reiterates along $x$ with a period $\Lambda$ controlled by the standing pump wave. For small $G_{1}$ the amplification maximum occurs in the center of the period. With increasing $G_{1}$ it splits spatially. Position of the transmission peaks is governed by the resonance condition $\delta_{20}+\left|G_{1}\right|^{2} \sin ^{2}(\pi x / \Lambda) / \delta_{2}=0$. The transmission behavior is associated with the ac-Stark shift of the Raman resonance induced by the pump field (see formula (A1)). As shown by Fig. 2a,c,e $|T(x)|$ depends on $G_{1}$ and $\delta_{20}$. The phase of the transmission function also exhibits a inhomogeneous distribution over the space period, depending on $G_{1}$ and $\delta_{20}$ (Fig. 2b,d,f).

Figures 3a,c,e illustrate the normalized Fraunhofer diffraction intensity according to the transmission function of Fig. 2. In the case of a Raman resonance $\delta_{20}=0$, diffraction into higher orders is very weak (Fig. 3a). When $\delta_{20} \neq 0$, under certain conditions the probe field is not only amplified in the zero-order direction but also distributes significantly into the high-order directions. Their intensity may be much more than the intensity of the probe field incident on the grating. That is, high-
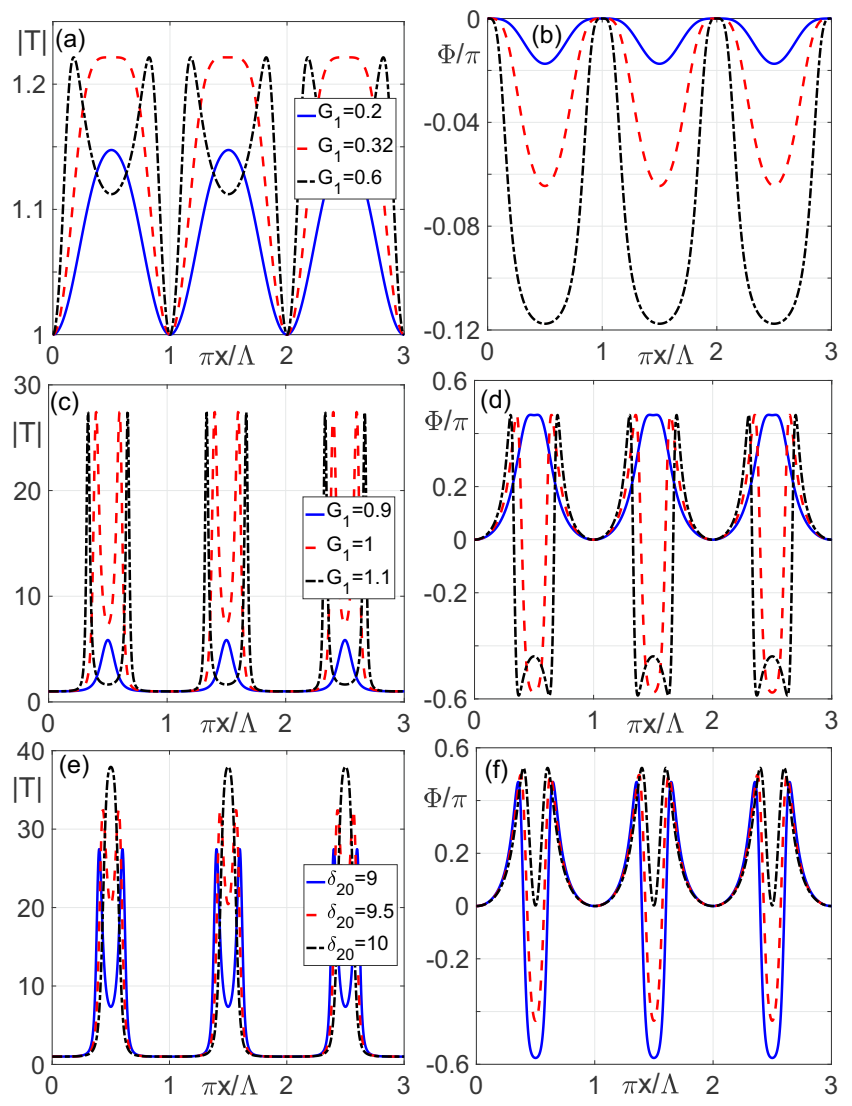

FIG. 2. The amplitude $|T(x)|$ (a,c,e) and the phase $\Phi(x)$ $(\mathrm{b}, \mathrm{d}, \mathrm{f})$ of transmission function as a function of $x$ over three space periods for various $G_{1}$ and $\delta_{20}$. (a,b) $\delta_{20}=0,(\mathrm{c}, \mathrm{d})$ $\delta_{20}=9$, and $(\mathrm{e}, \mathrm{f}) G_{1}=1$.

orders diffraction can also increase. The enhanced highorders diffraction appear at sufficiently high Raman gain of the probe field. The larger an amplification, the higher an intensity of high diffraction orders. For the given Raman detuning $\delta_{20}$, there is an optimal value of $G_{1}$ ensuring maximum diffraction peaks. The high-order diffraction modes may have sufficiently uniform intensity distribution (Fig. 3c). Thus, in contrast to the gratings based on EIT the RIDG is highly efficient in diffracting of light into high-order directions. Varying a pump field intensity and his frequency one can control the intensity of higher diffraction orders (Fig. 4). So, under certain conditions RIDG can be treated as a multi-beam-splitter with a controlled beam intensity.

Note that the RIDG is a hybrid grating being a combination of the amplitude (gain) and phase (refractive index) gratings. To illustrate the role of the amplitude and phase modulation, we show the Fraunhofer diffraction intensity for the amplitude grating (Fig. 3b,d,f), when the phase grating is not taken into account $(\Phi=0)$. It is seen that unlike conventional amplitude gratings, the amplitude RIDG distributes a light significantly into the high-order directions. This behavior is due to a combination of amplification and an inhomogeneous distribu- 

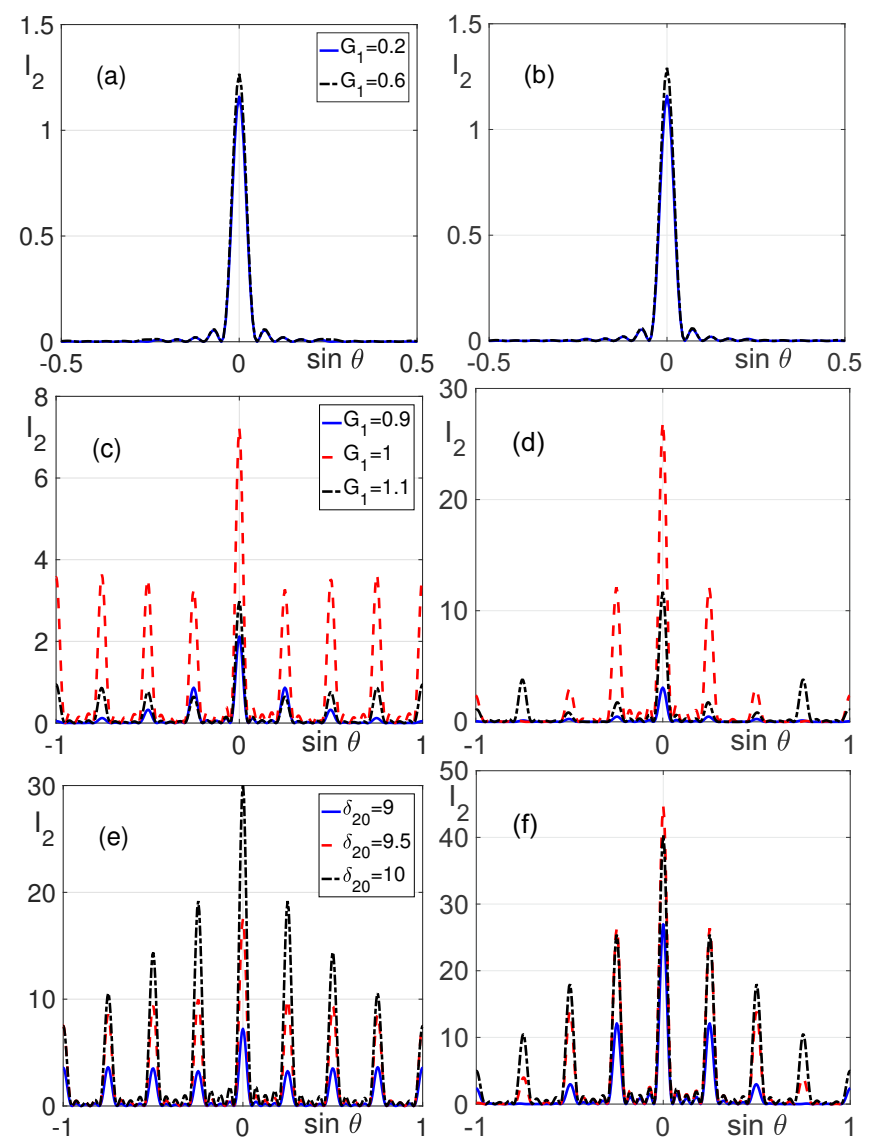

FIG. 3. (a,c,e) The Frauhofer diffraction intensity as a function of $\sin \theta$ for the transmission function $T(x)$ corresponding to Fig. 2. (b,d,f) The Frauhofer diffraction intensity as a function of $\sin \theta$ for the amplitude grating $|T(x)|$ corresponding to Fig. 2a,c,e.
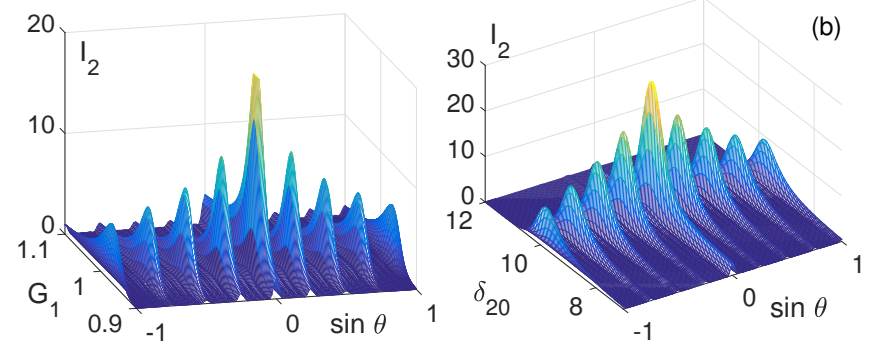

FIG. 4. The diffraction patterns $I_{2}(\sin \theta)$ as a function of $G_{1}$ when $\delta_{20}=9$ (a) and (b) $\delta_{20}=9$ when $G_{1}=1$. Diffraction patterns on an amplitude grating when phase modulation is ignored $\left(\operatorname{Re} F\left(\omega_{2}\right)=0\right)$ b) Full diffraction patterns on a hybrid grating. $L=40 . \delta_{1}=-100$

tion of the probe field at a grating period. Comparison of Fig. 3c,e and Fig. 3d,e shows that the diffraction intensity $I_{2}(\theta)$ is the resulting distribution including the diffraction effects from both amplitude and phase modulation. Although the phase grating is not ideal, it can substantially change quantitatively the diffraction pattern of the amplitude grating.
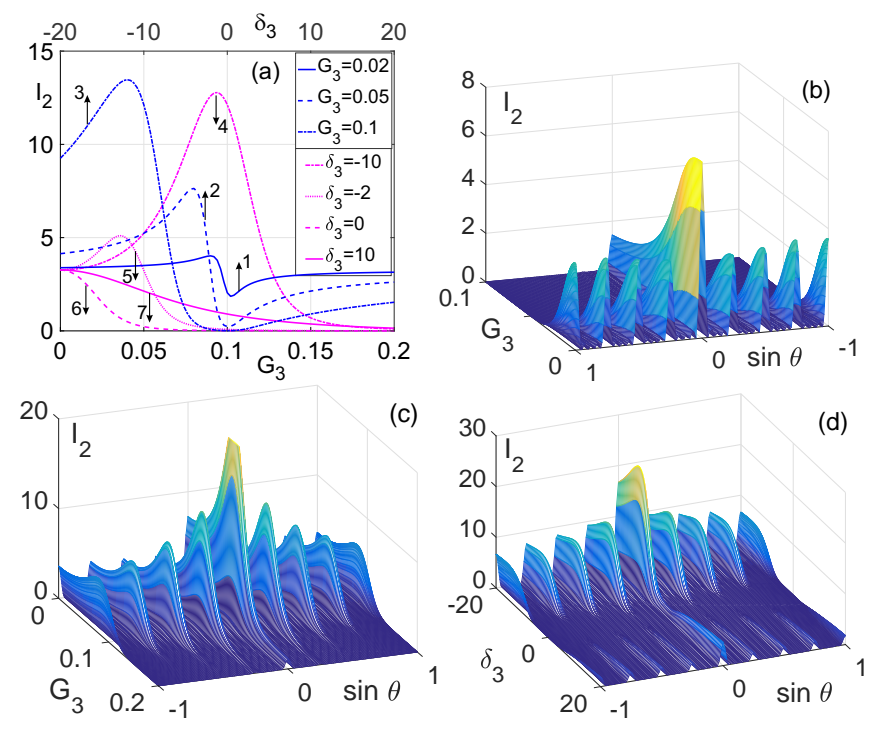

FIG. 5. (a) The first-order diffraction intensity $I_{2}\left(\theta_{1}\right)$ as a function of $\delta_{3}$ (for various $G_{3}$, curves 1-3) and $G_{3}$ (for various $\delta_{3}$, curves $4-7$ ). (b) The intensity of diffraction orders $I_{2}$ as a function of $G_{3}$ and $\sin \theta, \delta_{3}=0$. (c) $I_{2}$ as a function of $G_{3}$ and $\sin \theta, \delta_{3}=-10$. (d) $I_{2}$ as a function of $\delta_{3}$ and $\sin \theta$, $G_{3}=0.1$. Other parameters are $G_{1}=1, \delta_{20}=9$.

The use of an additional control field $E_{3}$ interacting with transition $|2\rangle-|3\rangle$ (Fig. 1a) opens up new possibilities for controlling the diffraction orders. In Fig. 5a, the diffraction efficiencies in the first-order direction are given as a function of the detuning $\delta_{3}$ (for various $G_{3}$, curves $1-3$ ) and $G_{3}$ (for various detunings $\delta_{3}$, curves $4-7$ ). It can be seen that $I_{2}$ strongly depends on both $G_{3}$ (under fixed $\delta_{3}$ ), and $\delta_{3}$ (under fixed $G_{3}$ ). Such a behavior is due to combined influence of the ac-Stark shift of the level $|1\rangle$ under the pump field $G_{1}$ and the ac-Stark shift of the level $|2\rangle$ due to the control field $G_{3}$. From Fig. 5a it can be seen that there are optimal parameters of the control field for which the first-order diffraction intensity is maximal. For certain values of the control field $\left(G_{3}\right.$ or $\delta_{3}$ ), the first diffraction order practically disappears. The high-orders diffraction behave in a similar manner (Fig. 5b,c,d). When the control field is resonant to the $|2\rangle-|3\rangle\left(\delta_{3}=0\right)$ transition, it suppresses diffraction into higher orders (Fig. 5b). A non-resonant control field may enhance the intensity of higher diffraction orders as well as suppress them (Fig. $5 \mathrm{c}, \mathrm{d}$ ). Maximum intensities are achievable at certain magnitudes of $G_{3}$, dependent on the initial Raman detuning: the larger the initial detuning, the greater the magnitude of $G_{3}$. Therefore, the intensity of diffraction orders can be controlled by varying the Rabi frequency of the control field or the control field frequency. Note that the intensity of the control field may be considerably lower than the pump field intensity. 

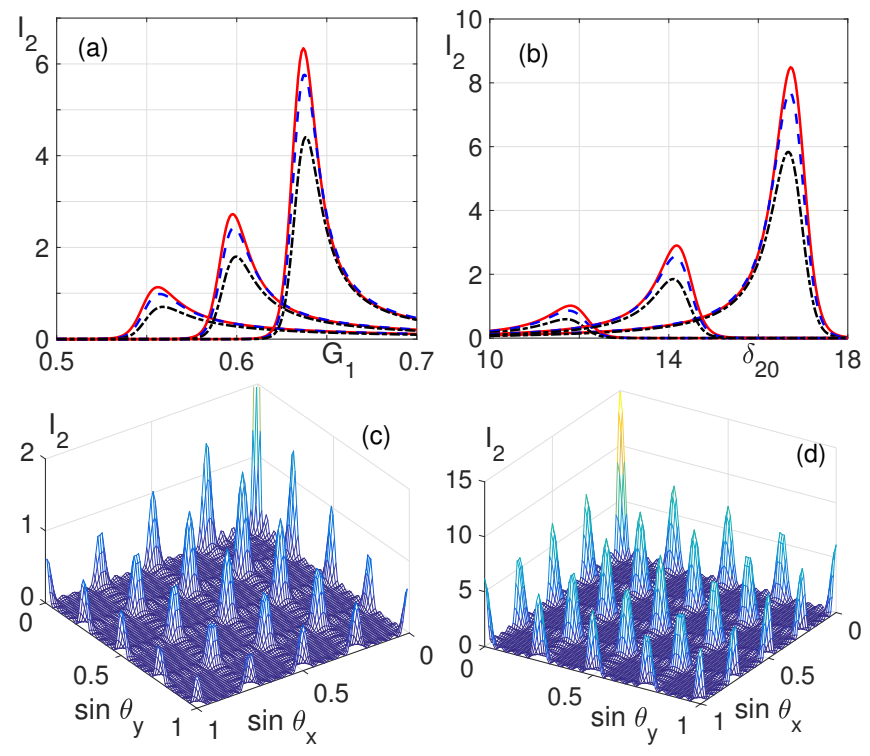

FIG. 6. (a) The diffraction intensity $I_{2}\left(\theta_{x}^{m}, \theta_{y}^{n}\right)$ as a function of $G_{1}$ at $\delta_{20}=12$ (left curves), 14 (middle), and 16 (right): $I_{2}\left(\theta_{x}^{1}, \theta_{y}^{3}\right)-$ solid, $I_{2}\left(\theta_{x}^{2}, \theta_{y}^{3}\right)-$ dash, $I_{2}\left(\theta_{x}^{3}, \theta_{y}^{3}\right)-$ dash dot. (b) The diffraction intensity $I_{2}\left(\theta_{x}^{m}, \theta_{y}^{n}\right)$ as a function of $\delta_{20}$ at $G_{1}=0.55$ (left), $G_{1}=0.6$ (middle), and $G_{1}=0.65$ (right): $I_{2}\left(\theta_{x}^{1}, \theta_{y}^{3}\right)-$ solid, $I_{2}\left(\theta_{x}^{2}, \theta_{y}^{3}\right)-$ dash, $I_{2}\left(\theta_{x}^{3}, \theta_{y}^{3}\right)-$ dash dot. (c, d) The Fraunhofer diffraction intensity $I_{2}\left(\theta_{x}, \theta_{y}\right)$ as a function of $\sin \left(\theta_{x}\right)$ and $\sin \left(\theta_{y}\right)$ at $G_{1}=0.55, \delta_{20}=11.4$ (c), and $G_{1}=$ $0.65, \delta_{20}=16.4(\mathrm{~d})$. A quarter of the full diffraction pattern is shown here.

\section{B. 2D RIDG}

Now consider a 2D RIDG. Such a grating can be realized when the pump field represents a superposition of two standing waves with amplitude $E_{1}$ perpendicular to each other (7). The Rabi frequency of the pump field is $G_{p}(x, y)=G_{1}\left[\sin \left(\pi x / \Lambda_{x}\right)+\sin \left(\pi y / \Lambda_{y}\right)\right]$. Further, we will assume that the $\Lambda_{x}=\Lambda_{y}=4 \lambda_{1}$. In order to obtain an effective high-order Fraunhofer diffraction in the present scheme, we demonstrate the diffraction intensity $I_{2}\left(\theta_{x}^{m}, \theta_{y}^{n}\right)$ dependence on the amplitude of the standingwave field $G_{1}$ (Fig. 6a) and Raman detuning $\delta_{20}$ (Fig. 6b). The interplay of several processes contributes to the outlined dependencies. Figure 6 a shows that $I_{2}\left(\theta_{x}^{m}, \theta_{y}^{n}\right)$ has maximum values subject to appropriate $\delta_{20}$. The larger the detuning $\delta_{20}$, the greater the pump field $G_{1}$ required to provide maximal gain of the probe field, and hence the greater resulting $I_{2}\left(\theta_{x}^{m}, \theta_{y}^{n}\right)$ at the maximum. The intensity $I_{2}\left(\theta_{x}^{m}, \theta_{y}^{n}\right)$ as a function of $\delta_{20}$ behaves in a similar manner (Fig. 6b). In Fig. 6c,d, we plot the Fraunhofer diffraction intensity as a function of $\sin \left(\theta_{x}\right)$ and $\sin \left(\theta_{y}\right)$ under different conditions. It can be seen that under certain conditions, all diffraction orders are amplified. It should be noted that the conditions for efficient generation of Fraunhofer patterns of high orders for 1D and 2D gratings are significantly different (see Figs. 3-4 and Fig. 6). We also note that even with a small amplifica-
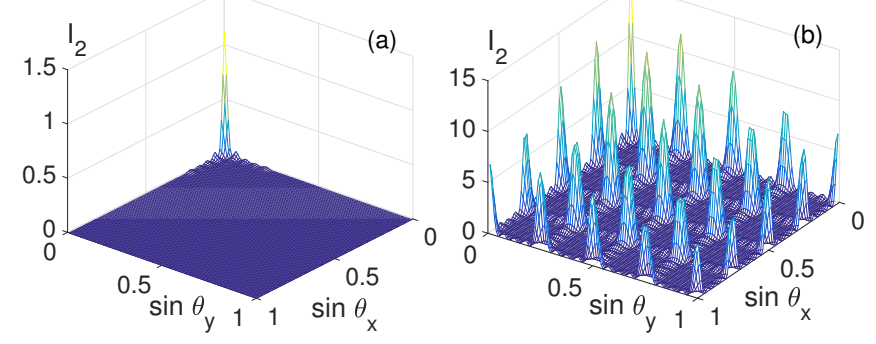

FIG. 7. The intensity of diffraction orders $I_{2}$ as a function of $\sin \theta_{x}$ and $\sin \theta_{y} . G_{1}=0.65, \delta_{20}=16.4, G_{3}=0.11$. (a) $\delta_{3}=0$, (b) $-\delta_{3}=-50$.

tion RIDG is more effective than the gratings based on EIT [25]. Using an additional laser field interacting with the $|2\rangle-|3\rangle$ transition, higher orders of diffraction can be controlled. For example, one can suppress higher orders of diffraction (Fig. 7a) or amplify them (Fig. 7b) under certain values of the intensity of the control field and its detuning $\delta_{3}$. So, under certain conditions 2D RIDG can be treated as a $2 \mathrm{D}$ multi-beam-splitter with controlled beam intensity.

\section{CONCLUSION}

We have theoretically investigated the diffraction characteristics of $1 \mathrm{D}$ and $2 \mathrm{D}$ atomic gratings based on periodic spatial modulation of a Raman gain and dispersion in three- and four-level atomic systems by using a standing-wave pump field, which is perpendicular to the probe wave. For a $2 \mathrm{D}$ grating, the pump field is a combination of two orthogonal standing-wave fields with the same frequency. It is shown that such gratings amplify not only the zero-order diffraction intensity but also under certain conditions all high-orders diffraction. So, RIDG is a amplifying multi-beam-splitter. In comparison with EIT-based gratings, the RIDGs have a much higher conversion efficiency of light in the high-orders directions. Diffraction of a probe field could be dynamically controlled by additional weak laser field choosing its detuning and strength. Using this field one can suppress or enhance the intensity of diffraction orders. These properties may give rise to such possible applications as optical switching, routing, beam deflectors and multi-beamsplitters for all-optical information processing.

\section{Appendix A}

In the appendix, we give the formula for the Raman susceptibility $\chi_{2}\left(\omega_{2}\right)$ in the cases when a control field is off $\left(G_{3}=0\right)$ and is on $\left(G_{3} \neq 0,\left|G_{1}\right|^{2} /\left|\delta_{1}\right|<\gamma_{20}\right)$. When $G_{3}=0$ and $\left|\delta_{1}\right| \gg \gamma_{10},\left|\delta_{2}\right| \gg \gamma_{12}$, formula for the 

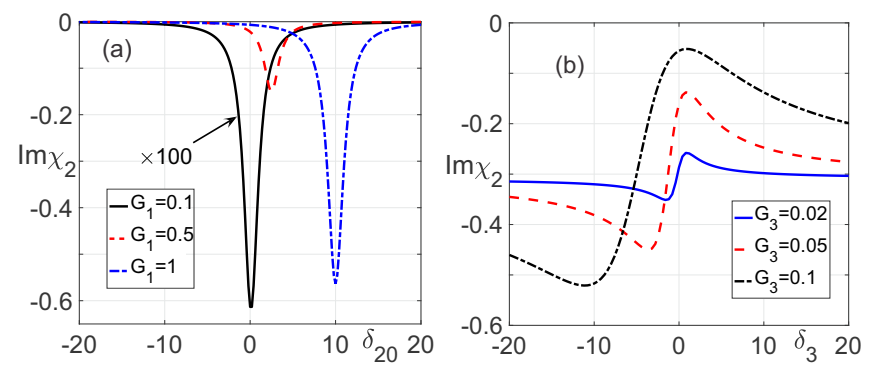

FIG. 8. (a) Variation of the imaginary part of the susceptibility $\chi_{2}$ with Raman detuning $\delta_{20}$ for different values $G_{1}$ in the absence of a control field $\left(G_{3}=0\right)$. (b) The imaginary part of the susceptibility $\chi_{2}$ as a function of $\delta_{3}$ in the presence of the control $\left(G_{3}\right)$ and pump $\left(G_{1}\right)$ fields, $\delta_{20}=9$. The common parameters are $G_{1}=1, \delta_{1}=-100$.

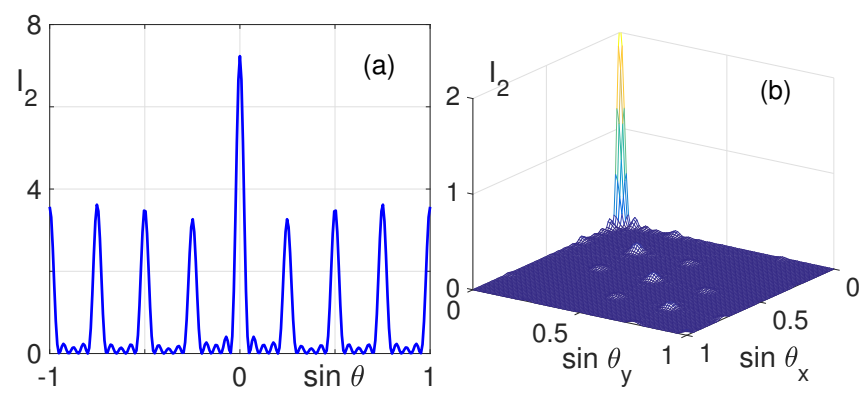

FIG. 9. The Fraunhofer diffraction patterns for the 1D (a) and $2 \mathrm{D}$ (b) gratings. $G_{1}=1, \delta_{20}=9, G_{3}=0, L=40$, $\delta_{1}=-100$.

susceptibility $\chi_{2}\left(\omega_{2}\right)$ (see $(1)$ and $\left.(2)\right)$ can be written as

$$
\chi_{2}\left(\omega_{2}, x\right)=\alpha_{r} \frac{\gamma_{12}\left|G_{1}\right|^{2} \sin ^{2}(\pi x / \Lambda)}{\delta_{1}\left[\left(\delta_{20}+i \gamma_{20}\right) \delta_{2}+\left|G_{1}\right|^{2} \sin ^{2}(\pi x / \Lambda)\right]} .
$$

When $\left|G_{1}\right|^{2} /\left|\delta_{1}\right|>\gamma_{20}$ the pump field creates an acStark shift of the state $|1\rangle$, which leads to a change in the probe detuning [35]. The magnitude of the ac-Stark shift $\delta_{1 S}=\left|G_{1}\right|^{2} \sin ^{2}(\pi x / \Lambda) / \delta_{1}$ depends on the transverse coordinate $x$. It is maximal at the antinode of the standing wave and decreases to zero at the nodes. Fig. 8a shows the behavior of $\operatorname{Im}\left(\chi_{2}\right)$ (a Raman gain) as a function of the Raman detuning $\delta_{20}$ for different values of $G_{1}$ when $G_{3}=0$. It can be seen that the Raman resonance shifts with increasing $G_{1}$. The amplitude of the perturbed resonance increases significantly in comparison with the unperturbed one.

When $\left|G_{1}\right|^{2} / \delta_{2} \ll \gamma_{20}$, expression (A1) can be written as

$$
\chi_{2}\left(\omega_{2}\right)=\chi_{R}\left(\omega_{2}\right) E_{1}^{2} \sin ^{2}(\pi x / \Lambda)
$$

where $\chi_{R}$ is the standard nonlinear Raman susceptibility [36].

In the case when $G_{3} \neq 0$ and the pump field is weak $\left(\left|G_{1}\right|^{2} /\left|\delta_{1}\right|<\gamma_{20}\right)$, the susceptibility can be expressed as $[37]$

$$
\chi_{2}\left(\omega_{2}, x\right)=-i \alpha_{r} \frac{\gamma_{12}}{\Delta_{1}} \frac{\left|G_{1}\right|^{2} \sin ^{2}(\pi x / \Lambda)\left(\Delta_{30} \Delta_{31}-\left|G_{3}\right|^{2}\right)}{\left(\Delta_{31} \Delta_{2}^{*}+\left|G_{3}\right|^{2}\right)\left(\Delta_{20} \Delta_{30}+\left|G_{3}\right|^{2}\right)} .
$$

Analysis (A3) shows that the off-resonant control field can induce an ac-Stark shift $\delta_{3 S}=\left|G_{3}\right|^{2} / \delta_{3}$ of the state $|2\rangle$, and the corresponding change in the probe detuning. In Fig. 8b, we demonstrate $\operatorname{Im}\left(\chi_{2}\right)$ as a function of a control field detuning for different Rabi frequencies $G_{3}$. In the general case, the spectral behavior of the susceptibility $\chi_{2}\left(\omega_{2}\right)$ is determined by the joint effect of the ac-Stark shifts due to the pump field and the control field. As a result, the largest gain modulation takes place when $\delta_{20}+\delta_{1 S}-\delta_{3 S} \approx 0$. Thus, the Raman amplification can be controlled both by the pump and control fields.

In the case of a 2D grating, the susceptibility (A1) has the form:

$$
\chi_{2}\left(\omega_{2}, x, y\right)=\alpha_{r} \frac{\gamma_{12}}{\delta_{1}} \frac{\left|G_{1}\right|^{2}\left[\sin ^{2}\left(\pi x / \Lambda_{x}\right)+\sin ^{2}\left(\pi y / \Lambda_{y}\right)\right]}{\left(\delta_{20}+i \gamma_{20}\right) \delta_{2}+\left|G_{1}\right|^{2}\left[\sin ^{2}\left(\pi x / \Lambda_{x}\right)+\sin ^{2}\left(\pi y / \Lambda_{y}\right)\right]}
$$

One can see that in the general case the $2 \mathrm{D}$ grating of the susceptibility is nonlinear in the pump field and cannot be regarded as a superposition of one-dimensional gratings in contrast to conventional gratings. Therefore, the optimal conditions for effective diffraction in the 2D grating will differ from the $1 \mathrm{D}$ gratings (see Fig. 9). It can be seen that for the selected parameters, the 1D grating has a gain in all orders, whereas in the 2D grating only the main maximum is amplified.

For a weak pump field, when the ac-Stark shift can be neglected, the $2 \mathrm{D}$ grating of the susceptibility is a superposition of one-dimensional gratings as follows from (A2). However, in this case, the amplification is small and therefore it is not of interest.
[1] M. Fleischhauer, A. Imamoglu, and J. P. Marangos, Rev. Mod. Phys. 77, 633 (2005).
[2] H. Y. Ling, Y.-Q. Li, and M. Xiao, Phys. Rev. A 57, 
1338 (1998).

[3] S.-q. Kuang, R.-g. Wan, P. Du, Y. Jiang, and J.-y. Gao, Opt. Express 16, 15455 (2008).

[4] J. Wen, Y.-H. Zhai, S. Du, and M. Xiao, Phys. Rev. A 82, 043814 (2010).

[5] A. W. Brown and M. Xiao, Opt. Lett. 30, 699 (2005).

[6] I.-H. Bae, H. S. Moon, M.-K. Kim, L. Lee, and J. B. Kim, Opt. Express 18, 1389 (2010).

[7] A. André and M. D. Lukin, Phys. Rev. Lett. 89, 143602 (2002).

[8] M. Artoni and G. C. La Rocca, Phys. Rev. Lett. 96, 073905 (2006).

[9] J.-H. Wu, M. Artoni, and G. C. L. Rocca, J. Opt. Soc. Am. B 25, 1840 (2008).

[10] M. Bajcsy, A. S. Zibrov, and M. D. Lukin, Nature 426, 638 (2003).

[11] J.-H. Wu, A. Raczyński, J. Zaremba, S. ZielińskaKaniasty, M. Artoni, and G. La Rocca, J. Mod. Opt. 56, 768 (2009).

[12] S. A. Moiseev, A. I. Sidorova, and B. S. Ham, Phys. Rev. A 89, 043802 (2014).

[13] C. Liu, S. Gong, D. Cheng, X. Fan, and Z. Xu, Phys. Rev. A 73, 025801 (2006).

[14] J. Wen, S. Du, H. Chen, and M. Xiao, Appl. Phys. Lett. 98, 081108 (2011), https://doi.org/10.1063/1.3559610.

[15] T. Qiu, G. Yang, and Q. Bian, EPL (Europhysics Letters) 101, 44004 (2013).

[16] Y. Zhang, C. Yuan, Y. Zhang, H. Zheng, H. Chen, C. Li, Z. Wang, and M. Xiao, Laser Phys. Lett. 10, 055406 (2013).

[17] V. G. Arkhipkin, S. A. Myslivets, and P. S. Pankin, Memoirs of the Faculty of Physics (2016).

[18] J. Tabosa, A. Lezama, and G. Cardoso, Opt. Commun. 165, 59 (1999).
[19] B. K. Dutta and P. K. Mahapatra, J. Phys. B: At. Mol. Opt. Phys. 39, 1145 (2006).

[20] H. Schmidt and A. Imamoglu, Opt. Lett. 21, 1936 (1996).

[21] L. E. E. de Araujo, Opt. Lett. 35, 977 (2010).

[22] S. A. Carvalho and L. E. E. de Araujo, Opt. Express 19, 1936 (2011).

[23] L. Zhao, W. Duan, and S. F. Yelin, Phys. Rev. A 84, 033806 (2011).

[24] L. Zhao, W. Duan, and S. F. Yelin, Phys. Rev. A 82, 013809 (2010).

[25] J. Wu and B. Ai, J. Phys. B: At. Mol. Opt. Phys. 48, 115504 (2015).

[26] L. Zhao, Scientific Reports 8, 3073 (2018).

[27] B. Xie, X. Cai, and Z.-H. Xiao, Optics Communications 285, 133 (2012).

[28] T. Naseri and R. Sadighi-Bonabi, J. Opt. Soc. Am. B 31, 2430 (2014).

[29] S.-q. Kuang, C.-s. Jin, and C. Li, Phys. Rev. A 84, 033831 (2011)

[30] L. Wang, F.-X. Zhou, H.-J. Guo, Y.-P. Niu, and S.-Q. Gong, Chinese Phys. B 25, 114205 (2016).

[31] V. G. Arkhipkin and S. A. Myslivets, Opt. Lett. 39, 3223 (2014).

[32] V. G. Arkhipkin and S. A. Myslivets, Phys. Rev. A 93, 013810 (2016).

[33] V. G. Arkhipkin and S. A. Myslivets, J. Opt. 19, 055501 (2017).

[34] A. K. Popov and S. A. Myslivets, Quantum Electron. 27, 1004 (1997).

[35] M. O. Scully and M. S. Zubairy, Quantum Optics (Cambridge University Press, 1997).

[36] R. W. Boyd, Nonlinear optics (London: Academic Press, 1992).

[37] G. S. Agarwal and S. Dasgupta, Phys. Rev. A 70, 023802 (2004). 